\title{
The role for S-carboxymethylcysteine (carbocisteine) in the management of chronic obstructive pulmonary disease
}

\section{Hooper \\ J Calvert}

North Bristol Lung Centre,

Southmead Hospital, Bristol, UK
Correspondence: James Calvert, MRCP, $\mathrm{MPH}, \mathrm{PhD}$

Consultant in Respiratory and Acute Medicine, Southmead Hospital, Westbury on Trym, Bristol BSIO 5NB, UK

Tel +44 I I 79505050

Email james.calvert@nbt.nhs.uk

\begin{abstract}
Prescription of mucoactive drugs for chronic obstructive pulmonary disease (COPD) is increasing. This development in clinical practice arises, at least in part, from a growing understanding of the important role that exacerbation frequency, systemic inflammation and oxidative stress play in the pathogenesis of respiratory disease. S-carboxymethylcysteine (carbocisteine) is the most frequently prescribed mucoactive agent for long-term COPD use in the UK. In addition to its mucoregulatory activity, carbocisteine exhibits free-radical scavenging and anti-inflammatory properties. These characteristics have stimulated interest in the potential that this and other mucoactive drugs may offer for modification of the disease processes present in COPD. This article reviews the pharmacology, in vivo and in vitro properties, and clinical trial evidence for carbocisteine in the context of guidelines for its use and the current understanding of the pathogenic processes that underlie COPD.
\end{abstract}

Keywords: carbocisteine, chronic obstructive pulmonary disease (COPD), exacerbation, free radicals, inflammation, mucolytic, oxidative stress, S-carboxymethylcysteine

\section{Introduction}

S-carboxymethylcysteine (carbocisteine or SCMC) is a mucoactive drug with in vitro free-radical scavenging and anti-inflammatory properties. Carbocisteine was first synthesized in the 1930s and became available for use as a mucoregulator in respiratory disease in the 1960s. A lack of consistency in data on clinical efficacy resulted in diminished use in the UK, and National Health System (NHS) formulary blacklisting in the 1980s. Throughout this time, prescribing of mucolytic/mucoactive agents for short- and long-term use remained common in Europe. However, a new understanding of the importance of exacerbation frequency in the progression of chronic obstructive pulmonary disease (COPD) and the role of systemic inflammation in the disease process, together with publication of several trials demonstrating positive effects of mucoactive drugs on exacerbation rates and quality of life, have rekindled interest in this family of drugs. There is increasing evidence that drugs commonly used for their mucoactive properties have diverse mechanisms of action, including significant anti-oxidant activity. In light of the well-established role of oxidative stress in the pathogenesis of COPD, it seems plausible that when these drugs are used over the long term they may modify the disease process. Carbocisteine became available by NHS prescription for chronic use in COPD once again in 2003.

\section{Carbocisteine: pharmacology}

Carbocisteine is available as an oral preparation both, as SCMC and its lysine salt (SCMC-Lys). The lysine group is cleaved on gastric absorption to form the active drug SCMC. Carbocisteine is a blocked thiol derivative of amino-acid; L-cysteine. 
The structure and mechanism of action of carbocisteine differs from other commonly available mucolytic drugs such as N-acetylcysteine (NAC) and erdosteine that bear free sulfhydryl (thiol) groups via which they split glycoprotein bonds in mucus.

Carbocisteine is well absorbed when taken orally, demonstrating one compartment open model kinetics thereafter. Peak serum concentrations are achieved at 1 to 1.7 hours and the plasma half-life is 1.33 hours. It achieves good penetration into lung tissue and bronchial secretions (Servin et al 1976; Braga et al 1982).

Approximately $30 \%$ to $60 \%$ of the drug is excreted unchanged in urine. Metabolic pathways are particularly complex with great inter-individual variation. They include acetylation, decarboxylation and sulfoxidation to produce inactive derivatives. Hepatic cytosolic sulfoxidation produces metabolites, which do not have the free-radical scavenging properties of the sulfide parent compound. Metabolism in this manner demonstrates a diurnal variation with less sulfoxidation occurring at night. There is also considerable variability in metabolism between individuals due to a genetic polymorphism in sulfoxidation capacity. Less efficient metabolism may result in increased exposure to the active parent compound and has been cited as one explanation for the inconsistent clinical response seen between individuals receiving the drug (Waring 1988; Steventon and Mitchell 2006).

There has been interest in the selection of patients for therapy on the basis of their metabolic activity for many years. In current practice, response is established on clinical grounds after a trial period, and non-responders are instructed to discontinue the drug. It is unclear whether those with seemingly appropriate clinical indications for carbocisteine who do not experience an improvement with its initiation are active sulfoxidizers and whether they might respond to a higher dose or nocturnal dosing regimens.

There is a theoretical risk of gastric erosion in the context of alterations in the characteristics of gastric mucus with all mucolytic agents. Carbocisteine is, therefore, contraindicated in active gastric ulceration. No significant toxicity has been reported either in animal models or following prolonged use in humans, and no drug interactions have been identified. Mild side-effects (largely gastric discomfort) are occasionally reported, eg, 1.8\% of patients in the treatment arm of one large study (Allegra et al 1996). Fixed drug eruption has also been infrequently associated with long-term prescribing of carbocisteine. It has been proposed that exposure to metabolic products, such as thiodiglycolic acid, may be responsible for this response. Thiodiglycolic acid is produced in greater concentration in poor sulfoxidizers, and so these reactions (ie, cutaneous) may be more common in this patient group (Steventon and Mitchell 2005).

Dose regimens for carbocisteine vary internationally, ranging from $750 \mathrm{mg}$ twice daily to $4.5 \mathrm{~g}$ once daily. There is little evidence to advocate one particular dosing regimen, although (as previously discussed) nocturnal administration may achieve higher concentrations of the active compound.

\section{Targets for carbocisteine in COPD: natural history and the role of oxidative stress}

COPD manifests as airway obstruction that is not fully reversible and deteriorates over time, at a rate greater than that seen with advancing age. The underlying disease process affects both the airways and interstitial lung tissue. Airway abnormalities include smooth muscle hypertrophy, mucus accumulation, wall thickening with neutrophil and inflammatory infiltrate, and an increase in number and size of goblet cells. The accompanying clinical syndrome of chronic bronchitis frequently co-exists with emphysema (the interstitial component) under the umbrella term of COPD. Central to the development and progression of the disease is a process of oxidative stress and proteinase-antiproteinase imbalance that frequently becomes self perpetuating (Repine 1997).

Oxidants derived from cigarette smoke and other environmental pollutants, in addition to those produced as a result of neutrophilic inflammation, inactivate antiproteinases (particularly $\alpha 1$-antitrypsin). This results in uncontrolled elastase activity and pulmonary tissue destruction, both by direct toxicity to protein and lipid structures and via the up-regulation of interleukin-8 (IL-8) activity. Elastase promotes the conversion of endogenous pulmonary xanthine dehydrogenase to xanthine oxidase, which acts on purine substrates and molecular oxygen to produce superoxide; a process that is central to oxidative stress in many inflammatory lung diseases.

Oxidants also exert a directly toxic effect on the structures of the lung, impair cilial activity and increase mucus production by goblet cells (Adler et al 1990; Feldman et al 1994).

Ongoing neutrophil and free-radical induced inflammation, along with the accumulation of tenacious mucus and airway 
remodeling with smooth muscle hyperplasia, results in increasing airflow limitation and creates an environment conducive to bacterial colonization. This, in itself, contributes to persistent inflammation and to neutrophil and alveolar macrophage recruitment to the airways, and is associated with a greater severity of airflow obstruction (Soler et al 1999; Zalacain et al 1999).

There is increasing evidence that COPD is a disease of systemic inflammation and that deterioration in parameters other than forced expiratory volume in one second $\left(\mathrm{FEV}_{1}\right)$ predict outcomes with greater sensitivity. Muscle dysfunction, both peripheral and diaphragmatic, is an important contributor to decline in patients with COPD. Quadricep strength and maximum inspiratory pressure generation have been shown to be predictors of mortality in moderate and severe COPD (Swallow et al 2007; Ottenheijm et al 2008). Elevated markers of oxidative stress have been demonstrated in the diaphragms of patients with severe COPD, and levels of protein oxidation have a negative correlation with respiratory muscle strength (Barreiro et al 2005). Peripheral muscles, particularly of the lower limbs, are also exposed to increased levels of both oxidative and nitrosative stress (Barreiro et al 2003). Both muscle groups demonstrate shifts in fiber type and atrophy that can be partially attributed to chronic inflammation and free-radical activity (Ottenheijm et al 2008).

Outcomes of relevance to disease progression in COPD have only been defined in recent years. Older studies tended to focus on lung function, a measure now thought to be a less useful marker of disease. Outcomes for the results of clinical interventions in COPD that are now thought to be relevant include: exacerbation rates; days of illness and hospital bed days; rate of lung function and exercise tolerance decline; all cause mortality; and improvement in health-related quality of life.

Acute exacerbations of COPD often occur in response to bacterial or viral infection and are recognized as deterioration in a patient's respiratory symptoms, frequently requiring additional treatment. Exacerbations incur significant healthcare costs and contribute to decline in patients' health-related quality of life (Seemungal et al 1998). Donaldson et al first established that more frequent exacerbations of COPD are associated with a greater rate of decline in lung function parameters (Donaldson et al 2002). It is recognized that some COPD patients experience frequent exacerbations, yet in others the disease takes a more stable course. Following many exacerbations, clinical and physiological state fails to return to baseline, and systemic inflammation continues on at a heightened level (Seemungal et al 2000).

\section{Clinical application of mucoactive drugs in COPD}

The 2006 Cochrane meta-analysis of mucolytic agents for COPD included 26 placebo-controlled, randomized clinical trials (RCTs) involving 7,335 patients (Poole and Black 2006). SCMC, SCMC-Lys and SCMC-sobrerol were included in only 3 of the trials, comprising a total of 486 subjects. The majority of the evidence included in the meta-analysis concerned NAC, the most widely used mucolytic agent internationally. The review concluded that there was evidence for a statistically significant reduction in the number of exacerbations per patient with long-term use (greater than 2 months) of oral mucolytics, of the order of $20 \%$ ( 0.5 exacerbations per patient per year). Overall, days of disability were reduced to a greater extent in the sub-set of intervention groups where this outcome was reported. Patients receiving mucolytics were more likely to experience no exacerbations than those receiving placebo, with a number needed to treat (NNT) of 6 . There is variability between the studies in reported changes in lung function parameters, which precludes a firm conclusion on the effect of mucolytics on spirometric parameters. Few of the studies included had measured health-related quality of life using validated tools and only one, concerning the use of erdosteine, demonstrated a significant improvement with the drug compared with placebo (Moretti et al 2004). The Cochrane review did not reveal a significant variation in outcome measures between different mucolytic agents and no serious adverse events were reported within the pooled data. The authors state that: "it is unlikely that the magnitude of the impact on exacerbation rates reported in the review will translate to COPD patients receiving current standard care". Firstly, it is assumed that many of the study patients will not have received inhaled corticosteroids (ICS), which were not in routine use in COPD prior to 1990, although this information is not specifically provided for many of the trials. Secondly, a great proportion of the studies were conducted over the winter period, during which exacerbation rates peak. The impact of mucolytic agents on the lower summer exacerbation rates is likely to be less impressive. The 2006 Cochrane review also raised the possibility of economic value in restricting mucolytic use exclusively to the winter months.

Supported by the National Institute for Health and Clinical Excellence (NICE) and British Thoracic Society (BTS) guidelines, mucolytic agents are currently in use 
selectively in COPD patients. The single available study on the cost-effectiveness analysis of mucolytics (specifically NAC) in COPD does not support prescribing in unselected patients with COPD (Grandjean et al 2000). At this time, there is a greater weight of evidence for their use in patients who exacerbate frequently and produce sputum chronically. Patients with significant symptoms related to sputum production, but who have mildly obstructive spirometry and therefore do not fulfil criteria for ICS, are likely to have the most to gain in terms of exacerbation reduction. This represents a small minority of COPD patients presenting to medical services. In practice, patient-reported improvement in symptoms related to sputum clearance and infective exacerbations is used to guide continuation with a mucolytic agent. In the clinical setting, most physicians are reluctant to temporarily suspend (ie, over the summer months) a safe intervention that has been associated with symptomatic relief.

Available mucolytic agents have been shown to have additional anti-inflammatory and anti-oxidant activities. In theory, therefore, they offer the potential to modify the disease, beyond aiding sputum clearance and reducing exacerbation rates. Clinical evidence to this effect would support their broader application in COPD. The Bronchitis Randomised on $\mathrm{N}$-acetylcysteine Cost-Utility Study (BRONCUS) sought to examine aspects of this hypothesis in an RCT comparing NAC $600 \mathrm{mg}$ daily with placebo, in 523 patients over a 3 -year period (Decramer et al 2005). The study, the longest of its kind, demonstrated no significant differences in lung function decline, exacerbation rates or deterioration in health status between patients in the treatment and placebo arms, except in the subgroup of patients not receiving ICS as standard management. No sufficiently long-term studies have been conducted with carbocisteine to specifically examine modification of disease progression or mortality as an outcome measure.

\section{In vitro, ex vivo and in vivo properties of carbocisteine Mucoregulation and effects on sputum clearance}

The viscoelastic properties of bronchial mucus are determined by glycoproteins (namely fucomucins, sialomucins and sulfomucins). Higher concentrations of fucomucins are found in the sputum of patients with COPD (Lamblin et al 1977).

Carbocisteine resets the balance between sialomucins and fucomucins (increasing concentrations of the former and reducing the latter) probably via the intracellular stimulation of sialyl transferase activity (Havez et al 1977). It is not thought to act on the structure of mucus once secreted into the airways in contrast to true mucolytic agents such as NAC. There is evidence from animal models that carbocisteine increases chloride transport across the airway epithelium and this may also contribute to its mucoregulatory action (Colombo et al 1994).

Several studies have demonstrated a reduction in measured sputum viscosity as early as 4 days after treatment initiation (Edwards et al 1976; Braga et al 1990). Braga et al examined the sputum viscosity and elasticity of 53 patients with chronic bronchitis when treated with $4.5 \mathrm{~g}$ of carbocisteine daily for 5 days compared with placebo (Braga et al 1989). There were significant reductions in both sputum characteristics with carbocisteine in patients with initial sputum viscosity and elasticity above the mean value previously established in similar patient groups. There is also in vivo and in vitro evidence of increased mucociliary clearance velocity with carbocisteine, particularly in patients with slow clearance before treatment (Kohler et al 1982; Braga et al 1990). This, apparently selective, action supports a mucoregulatory mechanism and, in clinical practice, supports the careful selection of patients for treatment with carbocisteine when it is prescribed for a possible symptomatic benefit associated with enhanced sputum clearance. Significantly, there are, also negative studies examining changes in sputum characteristics, in which different laboratory methods were employed (Thompson et al 1975; Goodman et al 1978). One explanation for this inconsistency is small study sample sizes combined with the inter-individual variability in response to the drug seen in clinical practice. However, there is also a lack of evidence correlating measurable mucoactive, drug-induced changes in sputum characteristics with clinical benefit. It is therefore not clear, when benefit is demonstrated, whether the mucoactive component to drug action should be credited. It should also be noted that in vitro studies of sputum viscosity are difficult to standardize and a commonly accepted methodology has not been derived.

There is evidence that carbocisteine increases the penetration of amoxicillin into bronchial secretions of patients with bacterial exacerbations of chronic bronchitis when the drugs are used concomitantly. This effect is probably due to the alterations in glycoprotein composition of sputum (Braga et al 1985). Improving antibiotic delivery to the site of bacterial infection may have a valuable effect, but the specific clinical implications of this finding on acute infective exacerbations have not been fully studied. 


\section{Actions on damaged epithelium and cough sensitivity}

Evidence from animal models of respiratory epithelial repair demonstrates that carbocisteine reverses neutral endopepsidase levels and reduces cough sensitivity (but not methacholine-induced bronchoconstriction) in rodents with antigen-damaged tracheal epithelium in a dose-related manner (Katayama et al 2001). Neutral endopeptidase is a cell membrane bound enzyme that modulates tachykinin-induced potentiation of: cholinergic motor transmission; smooth muscle contraction; mucus secretion; cough, and increases in vascular permeability in the airways. It has been repeatedly demonstrated in both human and animal studies that neutral endopeptidase levels are reduced in the presence of respiratory epithelial injury and associated bronchial hyper-responsiveness (Cheung et al 1992; Cheung et al 1993). Ishiura et al performed a double-blind, placebo-controlled, cross-over trial that illustrated a significant reduction in cough sensitivity to inhaled capsaicin (a C-fiber type bronchial cough receptor stimulator) in asthma patients following a 4-week course of carbocisteine (Ischiura et al 2003). The investigators proposed that an increase in neutral endopeptidase levels could explain this drug effect and that carbocisteine may have a role in the treatment of cough-variant asthma and other inflammatory airways disease in which intractable cough is a feature.

\section{Action on respiratory pathogens}

Attachment of pathogens to the respiratory epithelial cell surface is the first step in the development of acute respiratory tract infections, most of which are initiated by colonization of the pharynx.

One possible mechanism by which carbocisteine has the potential to reduce bacterial respiratory tract infections in COPD is the inhibition adherence of pathogenic bacteria to cells. In vitro studies have demonstrated a significant reduction in adherence of Moraxella catarrhalis to the pharyngeal epithelial cells, of both healthy subjects and those with chronic bronchitis treated with carbocisteine, when compared with placebo (Zheng et al 1999). Similarly, attachment of Streptococcus pneumoniae to pharyngeal epithelial cells taken from healthy subjects has been shown to be reduced by direct in vitro treatment of the cells with carbocisteine (Cakan et al 2003). It has been proposed that disruption of bacterial receptor sites on the epithelial surface and within mucus may explain these findings.

Rhinoviruses are associated with exacerbations of COPD. They increase oxidative stress and the production of inflammatory mediators. Intercellular adhesion molecule-1 (ICAM-1) is required for entry of rhinovirus ribonucleic acid (RNA) into cellular endosomes. Circulating ICAM-1 is present in greater concentrations in COPD and may have a role in neutrophil recruitment and retention in the lung, in addition to increasing susceptibility to viral respiratory tract infections (Riise et al 1994). Carbocisteine reduces expression of ICAM-1 and has been associated with a reduction in frequency of common colds and associated exacerbations in COPD patients (Tatsumi 2007).

\section{Anti-oxidant and anti-inflammatory activity}

As discussed, airway inflammation and oxidative stress due to inflammation, infection, inhaled environmental pollutants and (particularly) cigarette smoke plays a significant part in the development and progression of COPD.

Animal studies have demonstrated the anti-inflammatory action of carbocisteine in models of induced pulmonary inflammation involving several different cytokine profiles. Oral pre-treatment with SCMC-Lys appears to attenuate neutrophil recruitment in acute IL-1 $\beta$-induced airway inflammation and neutrophil, macrophage and eosinophil migration to the pleural space in carrageenan induced pleurisy (Asti et al 1995). Administration of carbocisteine to rats with sulfur dioxide-induced airway inflammation attenuates the secretion of abnormal mucous glycoproteins and reduces inflammatory cells, free radical and elastase activity in their bronchoalveolar lavage (BAL) fluid (Ishibashi et al 2001).

Carbocisteine has been shown to have relevant in vitro free-radical scavenging properties. NAC is recognized as a mucolytic agent with anti-oxidant behavior that is exerted via its free thiol group to form NAC disulfide. In contrast, carbocisteine does not carry a free thiol group but its thioether group can be oxidized by reactive oxygen species to form the sulfoxide by-product; however its anti-oxidant mechanism is not yet fully understood. Pinamonti et al conducted a series of experiments in 3 oxygen radical-producing systems (Pinamonti et al 2001). The experiments demonstrated that the addition of carbocisteine to the BAL fluid of stable COPD patients markedly reduced lymphocyte DNA destruction and reduced oxidative activity (measured as reduced cytochrome c concentration) of the solution in a dose-dependent manner. Free radical damage to deoxyribonucleic acid (DNA) in ultrasound-treated human serum was also reduced by the addition of carbocisteine. Finally, the concentration of xanthine oxidase products (superoxide and uric acid) 
in the supernatant of endothelial cells cultured with elastase was significantly reduced by the addition of carbocisteine to the culture.

Activated neutrophils produce a range of free radicals, with hydroxyl radical $\left(\mathrm{OH}^{-}\right)$and hypochlorus acid $(\mathrm{HOCl})$ being the most potent in terms of tissue injury. $\mathrm{HOCl}$ in particular oxidizes the methionyl residues of $\alpha 1$-antitrypsin, inactivating it and resulting in uncontrolled neutrophil elastase action. Intracellular production of $\mathrm{OH}^{-}$is implicated in IL-8 production that, in turn, has a role in the up-regulation of protease activity (Deforge et al 1992). Brandolini et al examined the free-radical scavenging capacity and its mechanism of SCMC-Lys in cell-free and cellular solutions (Brandolini et al 2003). In the cell-free studies, SCMC-lys demonstrated a potent, concentrationdependent reducing effect on $\mathrm{NaOCl}$ and $\mathrm{HOCl}$, with resultant oxidation of the drug's thioether group to form the sulfoxide $(\mathrm{S}-\mathrm{CMC}=\mathrm{O})$. In this model, the reducing capacity of SCMC-Lys was equal to that of glutathione (the chief endogenous anti-oxidant). A SCMC-Lys concentration-dependent reduction of $\mathrm{HOCl}$-mediated inhibition of $\alpha 1$-antitrypsin was also demonstrated. In the cellular studies, an equally potent free-radical scavenging action was demonstrated with $\mathrm{OH}^{-}$when a solution containing neutrophils and deoxyribose was combined with SCMC-Lys, which inhibited the selective oxidation of deoxyribose by neutrophil hydroxyl radicals. Finally, SCMC-Lys was shown to inhibit both paraquat- and IL- $1 \beta$-induced IL- 8 production by human peripheral blood mononuclear cells.

8 -isoprostane is produced by the reaction of oxygen radicals with arachidonic acid and other endogenous polyunsaturated fatty acids. It can be measured in breath condensate as a marker of oxidative stress in the lung. Exhaled 8-isoprostane is significantly elevated in stable, and to a greater extent exacerbating COPD (Montuschi et al 2000; Carpagnano et al 2004). Carpagnano et al demonstrated a significant reduction in both exhaled 8-isoprostane and pro-inflammatory cytokine, IL-6, in patients with COPD treated with SCMC-Lys for a 6-month period, providing an in vivo translation for the wealth of in vitro evidence that the drug has anti-inflammatory and anti-oxidant properties.

\section{Long-term use of carbocisteine and SCMC-Lys in COPD}

Clinical trial data on the use of carbocistiene in COPD are summarized in Table 1.
Several small studies conducted prior to 1990 compared $2.25 \mathrm{~g}$ to $3.00 \mathrm{~g}$ carbocisteine daily with placebo in chronic bronchitis, examining the outcome measures of lung function, sputum characteristics and symptom scores (Aylward 1974; Edwards et al 1976; Puchelle et al 1976; Grillage and Barnard-Jones 1985). Reported impact on lung function parameters is variable, but effect on the more subjective measures of cough and dyspnea resolution, and on increased sputum clearance is more consistently positive. These studies did not use well-validated outcome measures, and duration of carbocystiene administration varied from 10 days to 6 months, making it difficult to draw general conclusions about their clinical relevance.

Exacerbation rate has been demonstrated to have a significant impact on disease progression and health-related quality of life in COPD (Seemungal 1998). It therefore represents a relevant and definable outcome measure in clinical trials.

Four systematically-blinded and randomized studies comparing SCMC/SCMC-Lys with placebo and using exacerbations as the primary outcome measure have been reported. Allegra et al (1996) conducted their study over a 6-month period during the winter. Their 662 patients had baseline lung function suggestive of mild-to-moderate COPD. ICS were not excluded, but the proportion of patients in each group on ICS as standard treatment is not stated. The baseline $\mathrm{FEV}_{1}$ of the placebo group was significantly lower than that of the treatment group. Patients who received carbocisteine were significantly more likely to remain exacerbation free than the placebo group ( $\left.\chi^{2} 13.98, p=0.001\right)$. A large proportion of all study patients remained exacerbation-free $(54.0 \%$ in the placebo group compared with $70.4 \%$ in the continuous SCMC-Lys treatment group). Mean time to first exacerbation was significantly prolonged in the treatment group (96 days compared with 130 days). There was a statistically significant reduction in mean days of acute respiratory illness per patient $(3.8 \pm 7.4$ days compared with $6.7 \pm$ 9.7 days) with an equivalent reduction in days of antibiotics required. There were no statistically significant effects of intermittent treatment with carbocisteine compared with results in patients receiving placebo. The total number of exacerbations during the study period for each group was not reported (Allegra et al 1996).

An earlier double-blind, parallel-group study carried out in the UK examined the impact of $750 \mathrm{mg}$ carbocisteine 3 times daily compared with placebo on peak flow and exacerbation rate in 109 patients with chronic bronchitis 
(Medical Research Council defined) over 6 winter months (Grillage and Barnard-Jones 1985). The baseline characteristics of the groups are reported to be well matched, although no specific baseline statistics (including drug therapy at trial entrance) are listed. There was no demonstrable significant difference in exacerbation rate between treatment groups.

In a Japanese trial, Yasuda et al compared carbocisteine $1.5 \mathrm{~g}$ daily with placebo in 156 patients with COPD over a 12-month period (Yasuda et al 2006). The subjects tended to have mild COPD, and the 2 trial groups demonstrated no significant differences in baseline characteristics. Of note, no patients received ICS or oral corticosteroids during the study. There was a statistically significant reduction in the number of common colds (as defined by a validated 10 -point symptom scoring system) in the carbocisteine group (1.69 \pm 0.18 compared with $3.14 \pm 0.35$, relative risk [RR] 0.40 [95\% CI 0.20-0.80]). The exacerbation rate was also significantly reduced in the treatment group: RR 0.30 (95\% CI 0.20-0.70). Mean exacerbations per patient per year were $0.54 \pm 0.11$ in the treatment group compared with $1.38 \pm 0.20$ for placebo.

Most recently, the well-powered and systematically conducted Chinese PEACE study has demonstrated a clinically and statistically significant reduction in exacerbation rates in patients with moderate-to-severe COPD receiving carbocisteine over a 12-month period compared with placebo. A mean reduction of 0.34 exacerbations per patient per year was recorded in the treatment group (receiving $500 \mathrm{mg}$ carbocisteine 3 times a day for a 12 -month period) compared with placebo (Zheng et al 2008). Reduction in the rate of exacerbations only became significant after 6 months of treatment. Quality of life measures were also significantly improved at 12 months, consistent with the reduction in exacerbations. The study demonstrates the value of long-term carbocisteine in Chinese patients with COPD, but these results cannot be directly applied to European populations. Routine treatment for COPD in China includes the early prescription of methyl-xanthines and minimal use of ICS. In the study group, only $16.7 \%$ received ICS and, although this subgroup also demonstrated benefit from carbocisteine, it is likely that far lower-dose corticosteroids were in use than those routinely prescribed in Europe.

\section{Conclusions}

Carbocisteine exhibits a wide range of in vitro and in vivo anti-inflammatory and anti-oxidant activities in both human and animal models. In certain circumstances, it may diminish inflammatory cell recruitment to the airways, attenuate endothelial injury and associated cough sensitivity, and act as a potent, free-radical scavenger. These actions, in parallel with its mucoregulatory function, have theoretical relevance to pathogenic processes in COPD and other inflammatory airways disease. Clinical studies of carbocisteine in COPD examining the outcome measures of exacerbation rate and lung function parameters have produced heterogeneous results, reflecting those from studies with other mucolytic agents. Historically, trials have selected cohorts of patients with mild-to-moderate COPD, and there is circumstantial evidence that the impact of mucolytic and anti-oxidant agents is greater in patients who are not receiving ICS as standard management - an increasingly small group. There has not yet been a trial specifically focused on carbocisteine of adequate duration to answer questions about its direct impact on disease progression or survival in COPD. Results from the BRONCUS concerning NAC were inconclusive when these outcomes were considered (Decramer et al 2005).

Currently, the place for carbocisteine in COPD remains as a well-tolerated treatment with a favorable safety profile that provides symptomatic relief to some patients with sputum production, and that may reduce exacerbation rates via a range of mechanisms. Many uncertainties about carbocisteine remain. These include the relative clinical importance of its numerous mechanisms of action, appropriate patient selection and optimum dosing regimens (particularly to exploit the drug's antioxidant and anti-inflammatory activities). The majority of the evidence for carbocisteine's action is extrapolated from in vitro studies. To date, there are limited data from large randomized trials examining the effect of the addition of carbocisteine to existing treatment regimens, and so use of mucolytics is still largely based on clinical judgement and observation of symptomatic benefits in selected patients.

\section{Disclaimer and disclosures}

*This article has been supported by Teva UK Limited. Teva UK Limited have had the opportunity to comment on the medical content and accuracy of this article; however, final editorial control resides with the authors. The views and opinions of the authors are not necessarily those of Teva UK Limited.

The authors have no conflicts of interest to disclose. 
Table I Summary of some of the clinical trial data published on carbocisteine (SCMC)

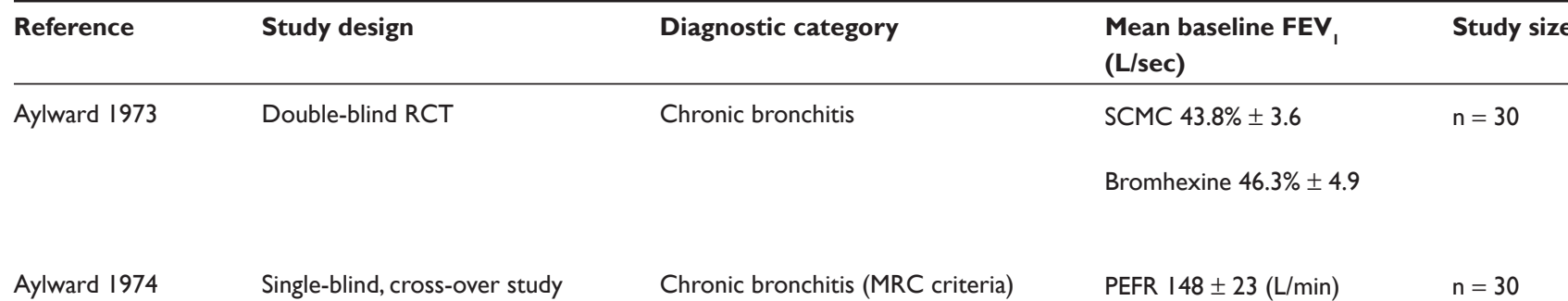

Placebo $45.1 \% \pm 15.23$ 


\begin{tabular}{|c|c|c|c|}
\hline Duration & Drug administration & Outcomes & Results \\
\hline \multirow[t]{4}{*}{10 days } & $750 \mathrm{mg}$ SCMC syrup tds vs bromhexine syrup & Increased sputum volume & $\mathrm{C}=\mathrm{B}$ \\
\hline & & & $\mathrm{C}=\mathrm{B}$ \\
\hline & & Increased sputum 'pourability' & \\
\hline & & $\mathrm{FEV}_{1} / \mathrm{PEFR}$ & $\mathrm{C}=\mathrm{B}$ (no change) \\
\hline \multirow[t]{5}{*}{3 months } & $15 \mathrm{~mL} 5 \%$ SCMC syrup tds vs placebo & Reduction in dyspnea & $C>p^{*}$ \\
\hline & & Sputum volume & $C>p^{*}$ \\
\hline & & Reduction in cough frequency and severity & $C>p^{*}$ \\
\hline & & Ease of expectoration & $C>p^{*}$ \\
\hline & & Improvement in incapacity & $C>p^{*}$ \\
\hline \multirow[t]{4}{*}{3 months } & 3 g SCMC syrup daily vs placebo & Sputum volume & $C>p^{*}$ \\
\hline & & Reduction in sputum viscosity & $C>p^{*}$ \\
\hline & & Reduction in dyspnea & $C>p^{*}$ \\
\hline & & Increase in $\mathrm{FEV}$ & $\mathrm{C}>\mathrm{p}^{* *}$ \\
\hline \multirow[t]{5}{*}{2 weeks } & I g SCMC tds vs placebo & Reduction in $\mathrm{FEV}_{1} / \mathrm{VC}$ ratio & $\mathrm{P}>\mathrm{C}^{* *}$ \\
\hline & & Other measures of airway obstruction & NS \\
\hline & & (MEFR $25 \%$ and $50 \%$ ) & \\
\hline & & Sputum viscosity (increased) & $C>p^{*}$ \\
\hline & & Improved clinical status & $C>p^{*}$ \\
\hline \multirow[t]{3}{*}{6 months } & $750 \mathrm{mg}$ SCMC tablets tds vs placebo & Increase in PEFR at $1,2,3$ and 5 months & $C>p^{*}$ \\
\hline & & Increase in PEFR at 4 and 6 months & NS \\
\hline & & Exacerbation rate & NS \\
\hline \multirow[t]{4}{*}{6 months } & $2.7 \mathrm{~g}$ SCMC daily vs placebo & Patients with no exacerbations & $C>p^{* * *}$ \\
\hline & & Time to first exacerbation & $C>p^{*}$ \\
\hline & & Mean days of acute respiratory illness & $\mathrm{C}<\mathrm{p}^{*}$ \\
\hline & & Mean days of antibiotic treatment & $\mathrm{C}<\mathrm{p}^{*}$ \\
\hline \multirow[t]{4}{*}{12 months } & $500 \mathrm{mg} \mathrm{SCMC}$ tds vs usual care & Number of common colds & $\mathrm{C}<\mathrm{p}^{* *}$ \\
\hline & & Number of exacerbations & $\mathrm{C}<\mathrm{P}^{* *}(\mathrm{RR} 0.37)$ \\
\hline & & Improvement in QoL (SGRQ) & $C>p^{* *}$ \\
\hline & & Change in $\mathrm{FEV}_{1}$ & $\begin{array}{l}C=p \\
\text { (no change) }\end{array}$ \\
\hline \multirow[t]{2}{*}{12 months } & $1.5 \mathrm{~g}$ SCMC daily vs placebo & Number of common colds & $\mathrm{C}<\mathrm{p}^{* *}$ \\
\hline & & Number of exacerbations & $\mathrm{C}<\mathrm{P}^{* *}(\mathrm{RR} 0.4)$ \\
\hline \multirow[t]{3}{*}{12 months } & $500 \mathrm{mg}$ SCMC tds vs placebo & Number of exacerbations & $\begin{array}{l}\mathrm{C}<\mathrm{p}^{* *} \\
\text { (risk ratio } 0.75 \text { ) }\end{array}$ \\
\hline & & Improvement in QoL (SGRQ) & $\mathrm{C}>\mathrm{p}^{* * *}$ \\
\hline & & Change in $\mathrm{FEV}_{1}$ & $\begin{array}{l}\mathrm{C}=\mathrm{P} \\
\text { (no change) }\end{array}$ \\
\hline
\end{tabular}

Abbreviations: C, carbocisteine (SCMC); Bm, bromhexine syrup; FEV „, forced expiratory volume in one second; MEFR, maximum expiratory flow rate; MRC, Medical Research Council; PEFR, peak expiratory flow rate; QoL, quality of life; RCT, randomized controlled trial; SGRQ, St George's Respiratory Questionnaire; tds, 3-times daily; VC, vital capacity; L/sec, liters per second; L/min, liters per minute.

Notes: ${ }^{\#}$ ebulized water produced equal results to SCMC. $p^{*}<0.05 ; p^{* *}<0.01 ; p^{* * *}<0.001$; NS, no statistically significant difference; †Inhaled or oral corticosteroids not permitted; $¥$ Oral corticosteroids at trial entry not permitted. $16.7 \%$ of trial patients on ICS. 


\section{References}

Adler KB, Holden-Stauffer WJ, Repine JE. 1990. Oxygen metabolites stimulate release of high molecular weight glycoconjugates by cell and organ cultures of rodent respiratory epithelium via an arachidonic acid - dependent mechanism. J Clin Invest, 85:75-85.

Allegra L, Cordaro C, Grassi C. 1996. Prevention of acute exacerbations of chronic obstructive bronchitis with carbocysteine lysine salt monohydrate: A multicentre, double-blind, placebo-controlled trial. Respiration, 63:174-80.

Asti C, Melillo G, Caselli G F, et al. 1995. Effectiveness of carbocysteine lysine salt monohydrate on models of airway inflammation and hyperresponsiveness, Pharmacol Res, 31:387-92.

Aylward M. 1973. A between-patient, double-blind comparison of S-carboxymethylcysteine and bromhexine in chronic obstructive bronchitis. Curr Med Res Opin, 1:219-27.

Aylward M. 1974. An assessment of S-carboxymethyl-cysteine in the treatment of chronic bronchitis. Curr Med Res Opin, 2:387-94

Barreiro E, Gea J, Corominas JM, et al. 2003. Nitric oxide synthases and protein oxidation in the quadriceps femoris of patients with chronic obstructive pulmonary disease. Am J Respir Cell Mol Biol, 29:771-8.

Barreiro E, de la PB, Minguella J, Corominas JM, et al. 2005. Oxidative stress and respiratory muscle dysfunction in severe chronic obstructive pulmonary disease. Am J Respir Crit Care Med, 171:1116-24.

Braga PC, Borsa M, De Anglis L, et al. 1982. Pharmacokinetic behaviour of S-carboxymethyl-cysteine-lys in patients with chronic bronchitis. Clin Ther, 4:480-8

Braga PC, Scaglione F, Scarpazza G, et al. 1985. Comparison between penetration of amoxicillin combined with carbocysteine and amoxicillin alone in pathological bronchial secretions and pulmonary tissue. Int $J$ Clin Pharm Res, 5:331-40.

Braga PC, Allegra L, Bossi R, et al. 1989. Identification of subpopulations of bronchitic patients for suitable therapy by a dynamic rheological test. Int J Clin Pharm Res, 9:175-82.

Braga PC, Allegra L, Rampoldi C, et al. 1990. Long-lasting effects on rheology and clearance of bronchial mucus after short term administration of high doses of carbocysteine lysine to patients with chronic bronchitis. Respiration, 57:353-8.

Brandolini L, Allegretti M, Berdini V, et al. 2003. Carbocysteine lysine salt monohydrate (SCMC-Lys) is a selective scavenger of reactive oxygen intermediates (ROIs). Eur Cytokine Netw, 14:20-6.

Cakan G, Turkoz M, Turan T, et al. 2003. S-carboxymethylcysteine inhibits the attachment of streptococcus pneumoniae to human pharyngeal epithelial cells. Microbial pathogenesis, 34:261-5.

Carpagnano GE, Resta O, Foschino-Barbaro MP, et al. 2004. Exhaled interleukine-6 and 8-isoprostane in chronic obstructive pulmonary disease: effect of carbocysteine lysine salt monohydrate (SCMC-Lys). Eur J Pharmacol, 505:169-75.

Cheung D, Bel EF, Hartigh JD, et al. 1992. The effect of an inhaled neutral endopeptidase inhibitor, thiorphan on airway responses to neurokinin A in normal human in vivo. Am Rev Respir Dis, 145:1275-80.

Cheung D, Timmers MC, Zwinderman AH, et al. 1993. Neural endopepidase activity and airway hyperreponsiveness to neurokinin A in asthmatic subjects in vivo. Am Rev Respir Dis, 148:1467-73.

Colombo B, Turconi P, Daffonchino L, et al. 1994. Stimulation of $\mathrm{Cl}^{-}$secretion by the mucoactive drug $\mathrm{S}$-carboxymethylcysteine lysine - salt in the isolated rabbit trachea. Eur Resp J, 7:1622-8.

Decramer M, Rutten-van Molken M, Dekhuijzen PN, et al. 2005. Effects of $\mathrm{N}$-acetylcysteine on outcomes in chronic obstructive pulmonary disease (BRONCHUS): a randomized placebo controlled trial. Lancet, $365: 1552-60$

DeForge LE, Fantone JC, Kenny JS, et al. 1992. Oxygen radical scavengers selectively inhibit interleukin 8 activity in human whole blood. $J$ Clin Invest, 90:2123-9.

Donaldson GC, Seemungal TAR, Bhowmik A, et al. 2002. Relationship between exacerbation frequency and lung function decline in chronic obstructive pulmonary disease. Thorax, 57:847-52.
Edwards GF, Steel AE, Scott JK, et al. 1976. S-carboxy-methyl-cysteine in the humidification of sputum and treatment of chronic airways disease. Chest, 70:505-13.

Feldman C, Anderson R, Kanthakumar K, et al. 1994. Oxidant mediated ciliary dysfunction. Free Radic Biol Med, 17:1-10.

Goodman RM, Yergin BM, Sackner MA. 1978. Effects of S-carboxymethlycysteine on Tracheal Mucus Velocity. Chest, 74:615-8.

Grandjean EM, Berthet PH, Ruffman R, et al. 2000. Cost effectiveness analysis of $\mathrm{N}$-acetylcysteine in chronic bronchopulmonary disease. Clin Ther, 22:209-20.

Grillage M, Barnard-Jones K. 1985. Long term oral carbocisteine therapy in patients with chronic bronchitis. A double blind trial with placebo control. The Br J Clin Pract, 39:395-8.

Havez R, Degand P, Roussel P, et al. 1977. Biochemical mechanism of action of cysteine derivatives on bronchial mucus. Poumon Coeur, 26:81-90.

Ischiura Y, Fujimura M, Yamamori C, et al. 2003. Effect of carbocysteine on cough reflex to capsaicin in asthmatic patients. Br J Clin Pharmacol, 55:504-10.

Ishibashi Y, Okamura T, Masumoto Y, et al. 2001. Effects of carbocysteine on airway inflammation and related events in $\mathrm{SO}_{2}$-exposed rats. Nihon Kokyuki Gakkai Zasshi, 39:17-23.

Katayama N, Fujimura M, Ueda A, et al. 2001. Effects of carbocysteine on antigen-induced increases in cough sensitivity and bronchial responsiveness in guinea pigs. J Pharmacol Exp Ther, 297:975-80.

Kohler D, Siebold A, Daikeler G. 1982. The influence of carbocysteine on mucociliary clearance. Atamweg - Lungenkrankankh, 8: 201-4.

Lamblin A, Humbert P, Degand P, et al. 1977. Heterogenicity of the sugar side chains of bronchial mucins isolated from two subjects with chronic bronchitis. Clin Chim Acta, 79:425-36.

Montuschi P, Collins JV, Ciabattoni G, et al. 2000. Exhaled 8-isoprostane as an in vivo biomarker of lung oxidative stress in patients with COPD and healthy smokers. Am J Respir Crit Care Med, 162:1175-7.

Moretti M, Bottrighi P, Dallari R, et al. 2004. The effect of long-term treatment with erdosteine on chronic obstructive pulmonary disease: the equalife study. Drugs Exp Clin Res, 30:143-52.

Ottenheijm CAC, Heunks LMA, Dekhuijzen RPN. 2008. Diaphragm adaptations in patients with COPD. Respir Res, 9:12.

Pinamonti S, Venturoli L, Leis M, et al. 2001. Antioxidant activity of carbocysteine lysine salt monohydrate. Panminerva Med, 43:215-20.

Poole PJ, Black PN. 2006. Mucolytic agents for chronic bronchitis or COPD. Cochrane Database of systematic reviews, Issue 3. Art No: CD001287.

Puchelle E, Girard F, Zahm ZM, 1976. Rheology of bronchial secretions and mucociliary transport. Bull Physiopathol Respir, 12:771-9.

Puchelle E, Aug F, Polu JM, 1978. Effect of the mucoregulator s-carboxymethyl-cysteine in patients with chronic bronchitis. Eur J Clin Pharmacol, 14:177-84.

Repine JE. 1997. Oxidative stress in chronic obstructive pulmonary disease. Am J Respir Crit Care Med, 156:341-57.

Riise GC, Larsson S, Lofdahl CG, et al. 1994. Circulating cell adhesion molecules in bronchial lavage and serum in COPD patients with chronic bronchitis. Eur Respir J, 7:1673-7.

Seemungal TAR, Donaldson GC, Paul EA, et al. 1998. Effect of exacerbation on quality of life in patients with chronic obstructive pulmonary disease. Am J Resp Crit Care Med, 157:418-22.

Seemungal TAR, Donaldson GC, Bhowmik A, et al. 2000. Time course and recovery of exacerbations in patients with chronic obstructive pulmonary disease. Am J Respir Crit Care Med, 161:1608-13.

Servin A, Garcet S, Huyen N, et al. 1976. Comparative pharmacokinetics of L-cysteine and one of its S-substituted derivatives, S-carboxymethylcysteine. J Pharmacol (Paris), 7:275-86.

Soler N, Ewig S, Torres A, et al. 1999. Airway inflammation and bronchial microbial patterns in patients with stable chronic obstructive pulmonary disease. Eur Respir J, 14:1015-22.

Steventon GB, Mitchell SC. 2005. Thiodiglycolic acid and dermatological reactions following S-carboxymethyl-1-cysteine administration. Br J Dermatol, 154:386-7. 
Steventon GB, Mitchell SC. 2006. The sulphoxidation of S-carboxymethyl-1cysteine in COPD. Eur Resp J, 27:865-6.

Swallow EB, Reyes D, Hopkinson NS, et al. 2007. Quadriceps strength predicts mortality in patients with moderate to severe chronic obstructive pulmonary disease. Thorax, 62:115-20.

Tatsumi K. 2007. Carbocysteine improves quality of life in patients with chronic obstructive pulmonary disease. $J$ Am Geriatr Soc, 55:1884-87.

Thompson ML, Pavia D, Jones CJ, et al. 1975. No demonstrable effect of S-carboxymethyl-cysteine on clearance of secretions from the human lung. Thorax, 30:669-73.

Waring RH. 1988. Pharmacogenetics of the S-oxidation of S-carboxymethylL-cysteine. Drug Metabol Drug Interact, 6:253-63.
Yasuda H, Yamaya M, Sasaki T, et al. 2006. Carbocysteine reduces frequency of common colds and exacerbations in patients with chronic obstructive pulmonary disease. J Am Geriatr Soc, 54:378-80.

Zalacain R, Sobradillo V, Amilibia J et al. 1999. Predisposing factors to bacterial colonization in chronic obstructive pulmonary disease. Eur Respir J, 13:343-8.

Zheng CH, Ahmed K, Rikitomi N, et al. 1999. The effects of Scarboxymethylcysteine and $\mathrm{N}$-acetylcysteine on the adherence of Moraxella catarrhalis to human pharyngeal epithelial cells. Microbiol Immunol, 43:107-13.

Zheng JP, Kang J, Huang SG et al. 2008. Effect of carbocisteine on acute exacerbations of chronic obstructive pulmonary disease (PEACE study): a randomised placebo-controlled study. Lancet, 371:2013-8. 
